\title{
Effect of Solar Tilt Angles on Photovoltaic Module Performance: A Behavioral Optimization Approach
}

\author{
T. Som ${ }^{1 *}$, A. Sharma ${ }^{1}$, D. Thakur ${ }^{2}$ \\ ${ }^{1}$ Department of Electrical \& Electronics Engineering, Dr. Akhilesh Das Gupta Institute of Engineering \& Technology, India \\ ${ }^{2}$ Department of Electronics \& Communication Engineering, Delhi Technical Campus, India \\ Email: trinasom@gmail.com
}

\begin{abstract}
In the present study, performance analyses of a solar module are made through the optimal variation of solar tilt angle, pertaining to the maximum generation of solar energy. The work has been carried out for a particular location at Tripura, in India, considering three different cases on an annual basis. An intelligent behavioural based algorithm, known as artificial bee algorithm (ABC), has been implemented for finding the optimal orientation of solar angle in analysing the performance. The result shows marginal differences are obtained in producing yearly maximum solar energy for different orientations of the PV module. It has been observed that the maximum average solar energy is obtained for the case where continuous adjustment is made by rotating the plane about the horizontal east-west axis within $20^{\circ}$ to $30^{\circ}$ tilt angle. The computed maximum and minimum of the monthly average efficiency is $10.9 \%$ and $8.7 \%$, respectively. Further, a comparative study has been performed in generating average solar energy through optimal tilt angle by the implementation of Perturb \& Observe method $(\mathrm{P} \& \mathrm{O})$. The monthly average solar power computed by $\mathrm{P} \& \mathrm{O}$ method resulted better in a range of $2 \%$ to $15 \%$ in comparison to that obtained by $\mathrm{ABC}$. While on the other hand, the efficiency computed by $\mathrm{ABC}$ algorithm was $15 \%$ to $19 \%$ better than that evaluated by $\mathrm{P} \& \mathrm{O}$ method for all the cases studied in the present work.

Keywords: solar power, tilt angle, optimization, artificial bee colony
\end{abstract}

\section{Nomenclature}

$\begin{array}{ll}P_{m p}: & \text { Maximum Solar Power Output } \\ V_{m p}: & \text { Voltage of Solar Module relating to maximum power } \\ V_{o c}: & \text { Open Circuit Voltage } \\ V_{o c, S T C}: & \text { Open circuit voltage at standard current } \\ \mu_{v o c}: & \text { Temperature co-efficient for determining Voc } \\ T: & \text { Operating Temperature on hourly basis } \\ T_{S T C}: & \text { Standard mean temperature } \\ I_{S C}: & \text { Short Circuit Current } \\ I_{S C}, \quad & \text { Short Circuit Current at standard mean setting } \\ G: & \text { Solar irradiance on hourly basis } \\ G_{S T C}: & \text { Standard Global radiation at a specific location } \\ \mu_{T S C}: & \text { Temperature co-efficient for determining Isc } \\ \eta: & \text { Instantaneous efficiency } \\ G A p v: & \text { Solar Intensity } \\ \delta: & \text { Declination angle } \\ n: & \text { Number of days in a year } \\ r_{s}: & \text { Solar Azimuthal Angle } \\ \alpha: & \text { Solar elevation angle } \\ \Phi: & \text { Latitude angle } \\ \Theta_{Z}: & \text { Zenith angle } \\ \beta: & \text { Tilt angle } \\ G_{T}: & \text { Total Solar irradiation }\end{array}$

Copyright $(2020$ T. Som, et al.

DOI: https://doi.org/10.37256/aie.122020505

This is an open-access article distributed under a CC BY license

(Creative Commons Attribution 4.0 International License)

https://creativecommons.org/licenses/by/4.0/ 


$\begin{array}{ll}G_{B}: & \text { Normal estimated component of solar irradiation } \\ G_{D}: & \text { Diffused component of solar irradiation } \\ G_{R}: & \text { Reflected component of solar irradiation } \\ G_{g}: & \text { Mean diffused radiation } \\ R_{b}: & \text { Ratio of daily average beam } \\ G_{d}: & \text { Sky diffused radiation } \\ \omega_{s s}: & \text { Sunset angle on hourly basis }\end{array}$

\section{Introduction}

Nowadays, as energy is a vital part of our life, it is essential for us to secure the supply and make it sustainable. The ever increasing energy requirement of the world consequently creates a lot of stress on traditional energy resources. Thus, the necessity for alternative energy sources has come up in order to provide sustainable power delivery systems ${ }^{[1]}$. Among various forms of non-conventional environment based energy sources, such as biomass, geothermal, wind, ocean, etc., solar energy has become one of the best prospects for widespread applications because of its accessibility and abundance ${ }^{[2]}$. The abundant solar energy is an obvious choice for clean energy, which can provide security for future growth and development ${ }^{[3]}$. Recently, many researchers have come up with several effective solar power generating approaches, in dealing with in-depth understanding of the operating principle of such systems ${ }^{[4]}$. Though, certain climatic conditions are best suited for the operation of solar modules, but, due to continuous unpredictable change in the weather, most solar photovoltaic units fail to operate in standard operating settings ${ }^{[5]}$ Lately, solar energy alternatives have gained significant attention through its most promising Photovoltaic power generation alternatives ${ }^{[6]}$. Further, to estimate the operational performance of different PV units in exposure to changes in climatic conditions, PV module's open-circuit voltage, short circuit current, and maximum power output along with efficiency has determined by authors in [7]. The solar unit's operational performance vary with and different environmental settings and the actual location of exposure ${ }^{[8]}$.

As, solar energy is very much required for its unpolluted, never-ending and bearable use, having many advantages ${ }^{[9]}$, so it is one of the best solutions which can deal and solve the fundamental energy crisis, along with green environmental issues ${ }^{[10-11]}$.

In many works of literature, the studies regarding efficiency of solar module with environmental parameters, like solar radiation, relative humidity and atmospheric temperature, and have been made [12]. Different renewable energy sources in terms of electric power generation have also been dealt with in [13]. Researchers in [14], showed various types of solar modules while considering various climates of different regions.

The tilt angle of a solar energy system is one of the important parameters for capturing maximum solar radiation falling on the solar panels. This angle is site-specific as it depends on the daily, monthly and yearly path of the sun.

Authors in [15] inferred that the performance of the solar module gets affected by the increase in solar irradiance incident on the PV module. The tilt angles of flat plate type PV module, are generally set to the local latitude of the specific place $^{[16]}$ in collecting the maximum yearly incident solar energy. Sometimes, the installation of PV plates with their optimum angles and orientation becomes hard due to the location and type of applications ${ }^{[17]}$. Hence, a study related to the performance of PV modules at different alignments of angle is of utmost importance. Fordham et al. compared the output of $50 \mathrm{~m}^{2}$ mono-crystalline silicon module relating to various tilt angle ${ }^{[18]}$. The power generated by a PV unit is based on many weather dependent factors, and thus a function of these parameters ${ }^{[19]}$. Several authors also calculated daily radiation for various slopes with different orientations, by the help of anisotropic model ${ }^{[20]}$. The study was performed in South Africa, resulting in $\beta$ opt and $\gamma$ opt. Many conventional and soft computing optimization techniques have been adopted by several researchers, in order to explore optimize solar power varying different parameters ${ }^{[21]}$.

Further, in order to accumulate solar radiation at maximum value for a particular time, the installation of PV panel should be made at an appropriate orientation of tilt angle considering respective circumstances ${ }^{[22]}$. The various circumstances which decide the orientation and installation for different solar positions are season, latitude of the particular place, and the time of the day ${ }^{[23]}$. Lately, many researchers, computed for optimal azimuth angle ( $\gamma$ opt) and optimum tilt angle $(\beta \mathrm{opt})$ for different solar collectors using different methods ${ }^{[24]}$. Moreover, in search of experimental hybrid techniques for calculating appropriate tilt angles of solar panel authors in [25] used ant direction hybrid differential evolution algorithm (ADHDEOA). However, the conventional methods, such as ergodic method takes infinite time to reach the optimal solution.

Hence, different types of meta-heuristic algorithms have been used in finding an optimal solution for tilt angle, while generating maximum solar power ${ }^{[26]}$. The gravitational search algorithm has been used for solar power generation through 
different orientations by authors in [27]. The evolution-based algorithms find their comfort in searching minimum value, while the MPPT techniques deliver better in finding maximum value. In consideration of both the traits, performance analyses of the two types of algorithm have been made in the present work.

Meta-heuristic algorithms prove to be competent in outperforming deterministic algorithms, especially when the complexity of the problem increases. Practitioners have utilized those unconventional algorithms for the past few decades. The $\mathrm{ABC}$ algorithm is a recently introduced population-based meta-heuristic optimization technique inspired by the intelligent foraging behavior of honeybee swarms.

The variation of tilt angle changes the amount of solar radiation that reaches the surface of the collector. Hence, the tilt angle is an important factor that affects the performance of a solar collector. In this study, a mathematical model is proposed for estimating the solar radiation on a tilted surface, which determines the optimum tilt angle of solar collector and its orientation (surface azimuth angle) in a specific period of time. The accurate determination of the optimum tilt angle for the location of interest is essential for maximum energy production by the system.

Though, many researchers have dealt with problems optimizing solar power generation through tilt angle position by soft computing methods; but this type of study in Indian scenario using behavioral optimization technique having an extensive foraging feature is not readily available from the literature.

Moreover, real-world optimization problems are very difficult and have high degrees of uncertainty. Conventional optimization algorithms have some limitations (i.e., local solution attainment and / or divergence) in solving such problems. On the other hand, meta-heuristic algorithms prove to be competent in outperforming deterministic algorithms, especially when the complexity of the problem increases. The artificial bee colony (ABC) algorithm is a population-based meta-heuristic optimization technique inspired by the intelligent foraging behavior of honeybee swarms, which does an extensive search by different types of bees.

This paper provides three typical types of control through single and continuous adjustment over tilt angle for determining the optimum position of the PV module pertaining to maximum power generation using $\mathrm{ABC}$ optimization technique.

\section{Problem formulation}

On the basis of climatological conditions of Tripura (India) over a typical year, maximum solar power generation has been computed for different tilt angle and orientation of a PV module. Three different case studies have been made in this regard. These are as follows;

Case 1: Monthly average solar power generation of a typical solar module; for the plane rotating about the horizontal east-west axis with single day adjustment.

Case 2: Monthly average solar power generation of a typical solar module; for the plane rotating about the horizontal east-west axis with continuous adjustment.

Case 3: Monthly average solar power generation of a typical solar module; for the plane rotating about north-south axis horizontally with continuous adjustment.

Mathematical Modeling

In the present work, the performance of $\mathrm{PV}$ modules has been analyzed, by calculated maximum power generation through variation of the tilt angle of the PV module. With the variation of tilt angle, different values of azimuthal angle, zenith angle, reflected component of solar irradiance, diffused component of solar irradiance, ratio of daily beam were determined. Further, using these values, short circuit current, open-circuit voltage, maximum voltage, maximum power, and efficiency were computed.

The following equations have been adopted. To calculate the dependence of short-circuit current Isc with irradiance and temperature ${ }^{[28]}$, we use equation (1):

$$
I_{s c}(G, T)=\left(\frac{G}{G_{s t c}}\right)_{1}\left[I_{s c, s t c}+\mu_{I s c}\left(T-T_{s t c}\right)\right]
$$

Again, for the dependence of the open-circuit voltage $V_{o c}$ as a function of temperature and irradiance and temperature coefficient; equation $(2)^{[10]}$ has been considered here

$$
V_{o c}(T)=\left[I_{o c, s t c}+\mu_{V_{o c}}\left(T-T_{s t c}\right)\right]
$$


The voltage relating to the maximum power (Vmp) and the maximum power of the PV modules has been evaluated from the following equation ${ }^{[28]}$ :

$$
\begin{aligned}
& V_{m p}=V_{o c}-V_{t} \ln \left\langle\left(V_{m p} / V_{t}\right)+1\right\rangle V \\
& P_{m p}=V_{m p} I_{m p}
\end{aligned}
$$

The calculation for the instantaneous efficiency corresponding to the maximum power output of the PV units is done by considering equation $(5)^{[28]}$

$$
\eta=P_{m p} / G A_{P V}
$$

The solar module parameters those have been considered here to calculate the Isc, Voc, Pmp, and $\eta$ are as follows:

1) Solar Angle: The latitude angle is the angle subtending the specific location and the center of the equator $(\Phi)$. The angle varies between $-90^{\circ}$ to $90^{\circ}$, considering north of the equator as positive and south as negative, as per convention.

The angle subtending between the sunlight of the arrays and the equator plane is known as declination angle $(\delta)$. It is generally evaluated by the given equation ${ }^{[29]}$

$$
\delta=23.45 \sin \left[360 *\left(\frac{284+n}{365}\right)\right]
$$

where $n$ is the number of days of the year. The azimuth angle is the compass direction from which the sunlight is coming.

The angle making between direct solar radiation and the position of the sun and the is known as solar azimuth angle $\left(\Upsilon_{S}\right)$, which is assumed to be negative towards east and positive towards west, when measured from south. Azimuth angle is computed by the equation shown below ${ }^{[29]}$.

$$
\Upsilon_{S}=\cos ^{-1}[(\sin (\alpha) \sin \Phi-\sin (\delta)) / \cos (\alpha) \cos \Phi]
$$

Further, the angle forming between the projections of the normal surface to that of the horizontal plane with is known as surface azimuth angle ( $\Upsilon$ ), which varies between $-180^{\circ}$ and $180^{\circ}$. The value of angle has been considered positive towards west, negative towards east and zero in south.

Zenith angle and solar elevation angle $(\alpha)$ are complementary functions. It can also be defined as the angle between the horizontal plane and the line to the sun. This elevation angle is calculated by the following equation.

$$
\alpha=90-\theta_{Z}
$$

$\theta_{Z}$ is the zenith angle between line and sun.

The tilt angle is the vital parameter of PV module in regard to produce optimum solar energy. Though the solar panels generates maximum energy, when the sun rays are vertical to the plane, but still the tilt angle is equal to the latitude of a specific place plus 15 degrees in winter or minus 15 degrees depending on the season ${ }^{[30]}$. Tilt angle $(\beta)$ varies from $0^{\circ}$ to $180^{\circ}$, and can be calculated considering different locations and adjustment of solar module. The tilt angle of PV cell is evaluated by rotating the plane about horizontal east-west axis for a single day adjustment ${ }^{[30]}$, as shown in equation below;

$$
\beta=\Phi-\delta
$$

Further, the tilt angle can be adjusted continuously, while rotating the plane about east-west horizontal axis. This can be expressed as given below ${ }^{[30]}$.

$$
\tan \beta=\tan \theta_{Z}\left|\cos \Upsilon_{S}\right|
$$

The third way of computing the tilt angle, is done by continuous adjustment where the plane is rotated about 
horizontal north-south axis. The equation is given below ${ }^{[30]}$.

$$
\tan \beta=\tan \theta_{Z}\left|\cos \Upsilon_{S}-\Upsilon_{S}\right|
$$

The summation of the total direct Global irradiance is generally is normal solar rays incident onto the horizontal surface, incident diffuse radiation and the reflected component of Solar Irradiation Hence, the incident total radiation for a given tilted surface, is given by the equation below ${ }^{[30]}$.

$$
G_{T}=G_{B}+G_{D}+G_{R}
$$

Where, $G_{R}$ is the reflected component of the radiation, $G_{T}$ is the average daily radiation on a monthly basis, $G_{B}$ is normally estimated radiation of the direct beam, and $G_{D}$ is the diffuse component of the radiation. Thus the total daily radiation received on daily basis for an inclined surface can be computed as ${ }^{[32]}$ :

$$
G_{B}=\left(G_{g}-G_{d}\right) R_{b}
$$

Where, $G_{d}, R_{b}$ and $G_{g}$ denotes mean diffuse radiation on daily basis, ratio of daily average beam radiation to that of a tilted surface on the same horizontal plane, and global radiation respectively. The function of reflected radiation at the ground can be written as ${ }^{[32]}$ :

$$
G_{R}=\left(\frac{1-\cos \beta}{2}\right) G_{g}
$$

As suggested by authors in [25], $R_{b}$ can be calculated; considering the slope towards the equator.

$$
R_{b}=\frac{\cos (\varnothing-\beta) \cos \delta \sin \omega_{S S}+\omega_{S S} \sin (\varnothing-\beta) \sin \delta}{\cos Ø \cos \delta \sin \omega_{s S}+\omega_{s S} \sin \varnothing \sin \delta}
$$

Where $\omega_{s s}$ is sunset angle on an hourly basis in the mean day of the month for any tilted surface. The sunset hour angle can be expressed as ${ }^{[33]}$

$$
\omega_{S S}=\operatorname{Arccos}(\tan (\varnothing) \tan \delta)
$$

The sky diffused radiation can be calculated as ${ }^{[31]}$

$$
G_{D}=\left(\frac{1+\cos \beta}{2}\right) G_{d}
$$

Considering all the geographically based location parameters, and known load data of the particular place, artificial bee colony $(\mathrm{ABC})$ algorithm has been employed for optimal solar power generation with variation in orientation and tilt angle of PV module. Artificial Bee Colony (ABC) algorithm is a swarm-based optimization algorithm with advantages like simplicity and proper exploration ability.

\section{Optimization technique}

Artificial Bee Colony Algorithm

Artificial bee colony algorithm (ABC) was first proposed by Karaboga and Basturk in $2005^{[34]}$. ABC a new swarmbased technique whose operation is based on the collective intelligent behavior of the honey bees. The performance of the $\mathrm{ABC}$ algorithm is better than or similar to those of other population-based algorithms with the advantage of employing fewer control parameters. The ABC algorithm also has the advantages of strong robustness, fast convergence and high flexibility.

It finds a great application in various types of optimization problems, like load balancing problem ${ }^{[35]}$; and optimal power flow ${ }^{[36]}$. 
At first, the employed bees search for the sources of food randomly and send the information to the onlooker bees. Then, depending on the information, the onlooker bees take a decision on whether or not to explore the food sources. The employed bee then starts foraging for a new food source for the unrestricted food source and becomes a scout ${ }^{[25]}$. Thus two types of bees help in searching the global minima instead of getting trapped in a locally optimal solution.

The proposed ABC algorithms have been implemented in calculating the optimum solar tilt angle for the abovementioned problem, and the working flow diagram has been shown below in figure 1 .

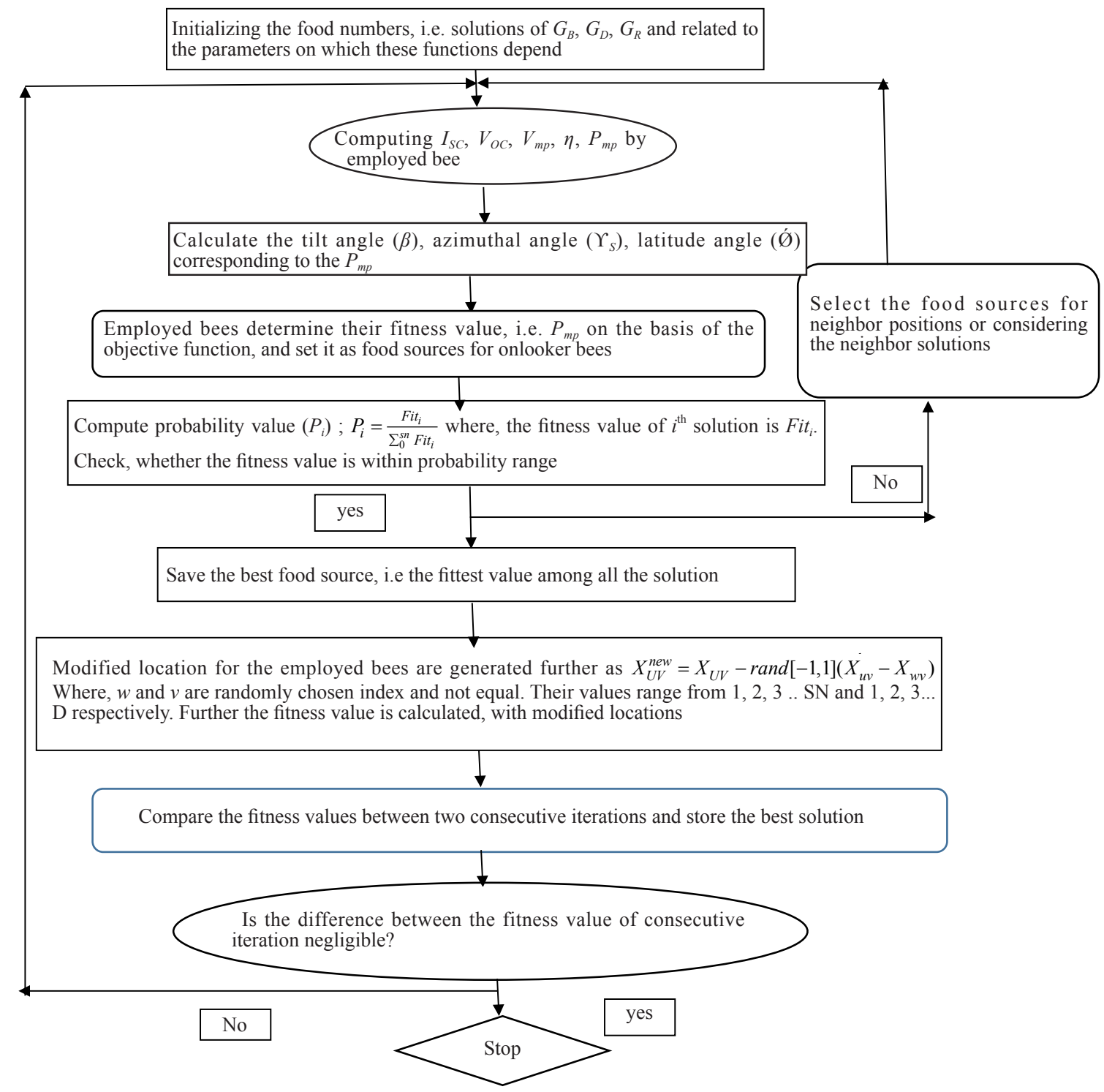

Figure 1. Flow diagram of Artificial Bee Colony Algorithm

\section{Numerical analyses}

Input parameters considered for performing the case studies in the given scenario are described below;

\subsection{Input data}

As India is a tropical country, hence the division of day and night is almost same throughout the year. Hence, to capitalize the maximum solar rays, the climatological and geographical conditions of India has been chosen for this study. Moreover, with increasing pollution in most densely populated cities, solar rays fail to reach solar panels. Thus the input data in the present work corresponds to a relatively clean city like Tripura. The input data for a typical year in terms of $G_{T}$, $\mathrm{W}$ and $\mathrm{Ta}^{[37]}$, are shown in table 1 and figure 2 and figure 3 , as follows; 


\begin{tabular}{cc} 
Table 1. Geographical data of tripura \\
\hline Country & India \\
Place & Tripura \\
Latitude & 23.745127 \\
Longitude & 91.746826 \\
\hline
\end{tabular}

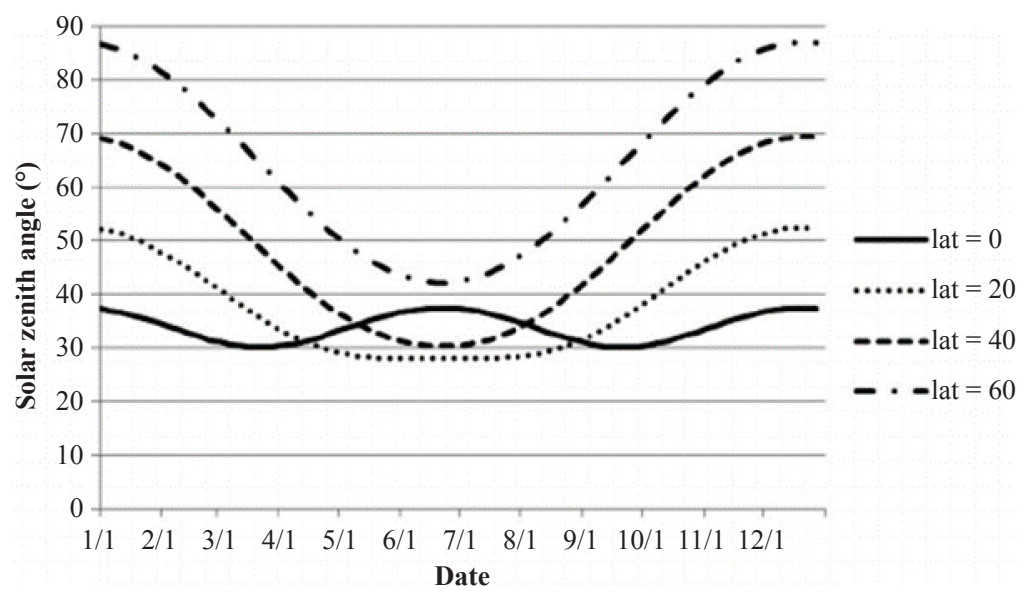

Figure 2. Relation of zenith angle with the latitude of a specific place

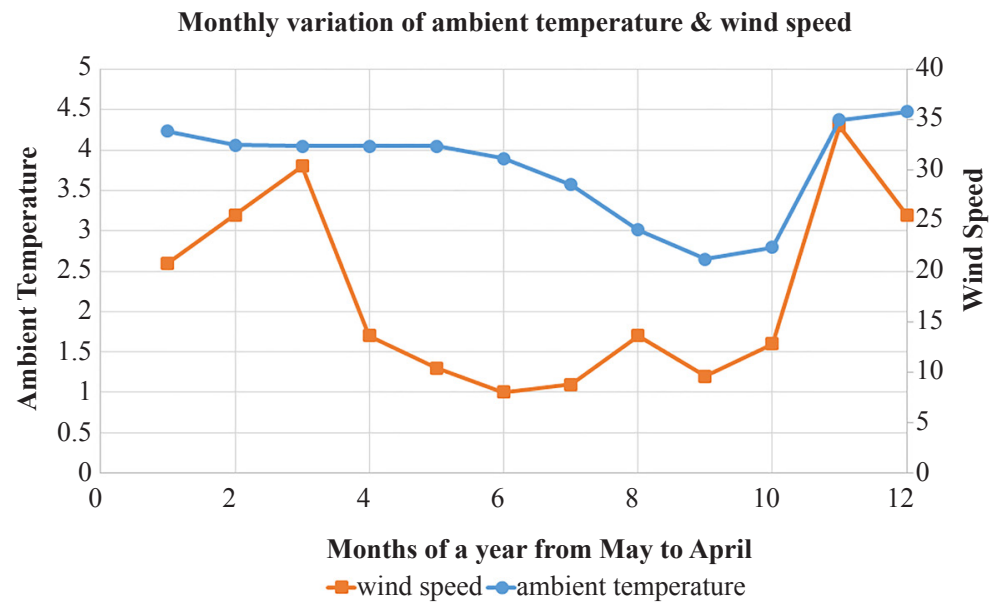

Figure 3. Variation of ambient temperature and wind-speed with efficiency, over a typical year in Tripura

Interference from pollution may be a limiting factor in realizing the potential of solar power in developing nations. Therefore a pollution-free place like the one studied in the manuscript can be an interesting case study.

Input Data for implementation of ABC Technique

Dimension / Element of the problem (D): 4

Employed Bees $=100$

Onlooker bees $=100$

Colony size (NP): $100 \times 4$

Solution or food number $=$ Half the number of colony

Maximum cycle / iteration: 1000

With all the objective functions and predefined parameters of computing technique, in house code for both the algorithms has been developed using M-File of Matlab, version 12. The PC configuration used is Intel i5 series CPU, 8 GB RAM, nominal clock-speed $1.65 \mathrm{GHz}$ on a personal computer. The programs had a runtime of the order of minutes.

\subsection{Results}

The specific test cases are performed with the real-time input data of a small Indian locality, in Tripura, and various results are obtained, which has been shown and discussed below. In case I, the single-day adjustment was made, on the 
basis of equation 9. Ideally, a fixed, roof-mounted solar energy system should be at an angle that is equal to the latitude of the location where it is installed, but sometimes anything between 30 to 45 degree works better. So, the authors here have set the single-day adjustment of tilt angle at 30,35, 40 and 45 degrees over 8 hours of daily solar irradiation received by the place. With this setting, $\mathrm{ABC}$ computed, values for azimuthal angle $\left(\Upsilon_{S}\right)$, zenith angle $\left(\theta_{Z}\right)$, normal estimated solar irradiance $\left(G_{B}\right)$, reflected component $\left(G_{R}\right)$, diffused component $\left(G_{D}\right)$, which are dependent on the tilt angle was calculated and further fed to the equations for calculating Isc (short circuit current), and Voc (open circuit voltage) to find out the maximum power generated as Pmp. A steady variation of monthly solar incident irradiance has been attained for case I, which shows relatively insignificant relation with the variation of tilt angle. Moreover, in comparison with case II and III, the average monthly solar energy is less than that obtained using case I.

Further, in case II, for the plane rotating about the horizontal east-west axis with continuous adjustment have been considered following equation 10. Besides the variation of tilt angle $(\beta)$ from 0 to 60 degree, the azimuthal angle $\left(\Upsilon_{S}\right)$ has been varied from -180 to 180 degree, considering positive towards the west and negative towards east.

Similarly,

Case III: Monthly average solar power generation of a typical solar module; for the plane rotating about north-south axis horizontally with continuous adjustment.

The variation of monthly solar irradiance incident on the solar module with respect to different tilt angles for case II has been portrayed in figure 4 .

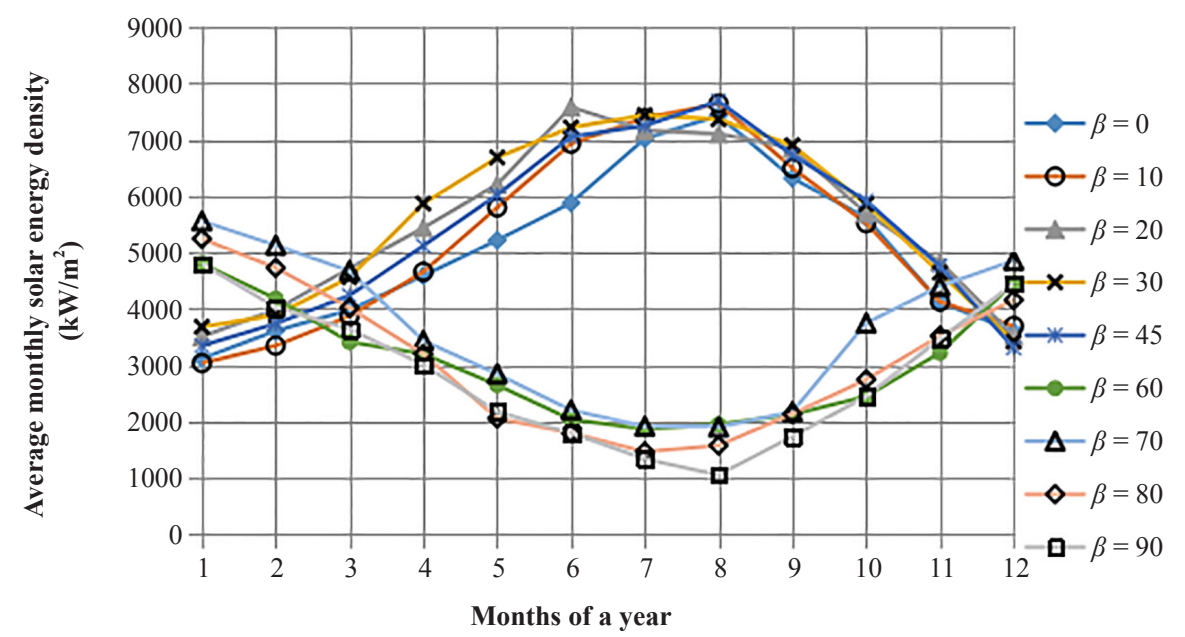

Figure 4. Variation of average monthly solar energy with different tilt angles for Case II as computed by ABC

A maximum and minimum values of the monthly average solar irradiance has been obtained as $6.7 \mathrm{kWh} / \mathrm{m}^{2}$ during the summer season, and $2.5 \mathrm{kWh} / \mathrm{m}^{2}$ during the winter season respectively.

In case III, continuous adjustment for the plane of the solar module horizontally rotating about north-south axis has been performed to generate solar power following equation 11. The latitude angle $(\Phi)$ also varies in this case from that of case II. Case III depicts the average energy output on a monthly basis for the PV modules, which varies with change in tilt angle as shown in figure 5 . 


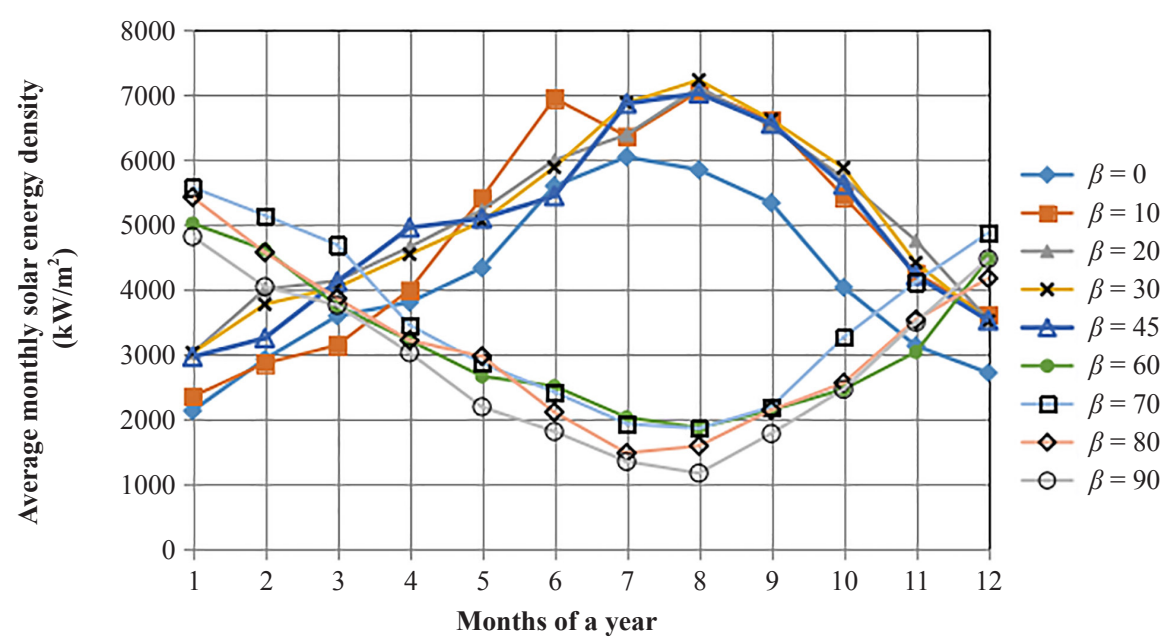

Figure 5. Variation of average monthly solar energy with different tilt angles for Case III as evaluated by ABC

The maximum average output energy on a monthly basis has found to have a similar pattern of variation as that obtained in case II. The maximum monthly average incident solar energy achieved was $5.52 \mathrm{kWh} / \mathrm{m}^{2}$ in winter and 2.0 $\mathrm{kWh} / \mathrm{m}^{2}$ in winter.

A typical representation of the variation of yearly average efficiency, as computed by $\mathrm{ABC}$ considering equation (5) with respect to different tilt angles for case III, have been presented in figure 6 . The curves obtained for efficiencies are found to have an opposite drift from that obtained for average output energy per month. Yearly maximum output energy has been achieved for case II when the tilt angle varies continuously from $20^{\circ}$ to $45^{\circ}$.

The surface temperature of the PV unit increases with the increase in solar incident energy and thereby decreasing the module efficiency. The results show a maximum value of $10.6 \%$ for case II; and minimum value of $9.1 \%$ in case III, when the tilt angles of the module have been varied over the range, shown in figure 5.

During the summer season, the efficiency on a monthly basis ranges from an $8.9 \%$ of a minimum value to $10.9 \%$ of maximum value as calculated for different tilt angles in case II. Efficiency obtained in autumn season is lower than that obtained in the spring season.

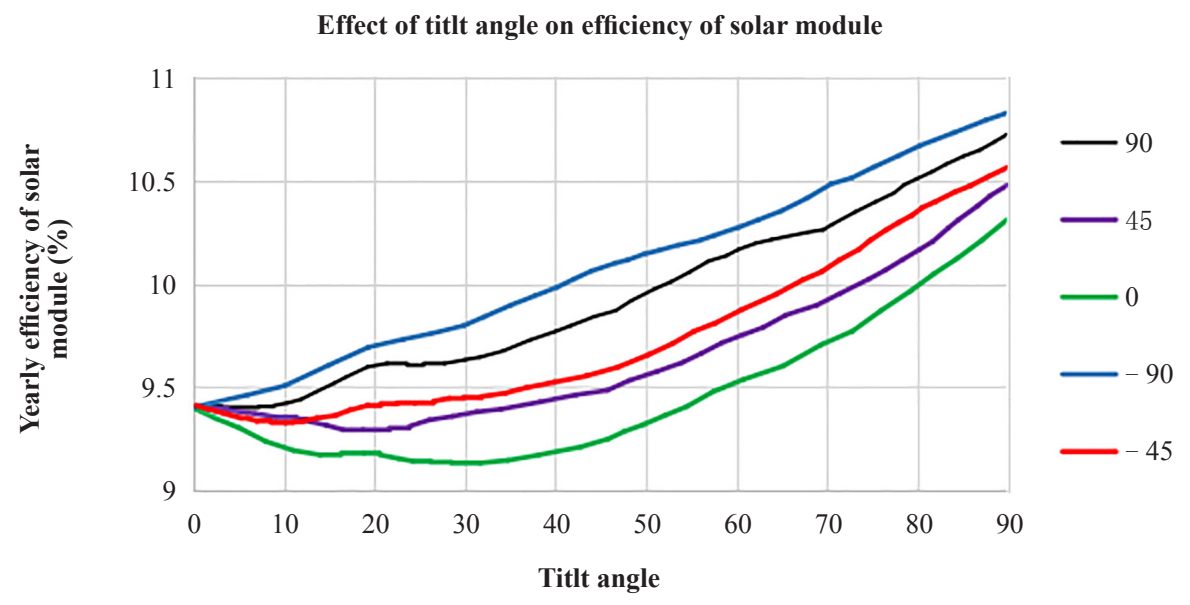

Figure 6. Variation of monthly average efficiency with respect to different tilt angles, for case III

Finally, it can be inferred that, in case II, horizontal PV modules yield more optimal values of yearly maximum output energy, than that yield for case III. Artificial bee colony computed results shows that PV modules yield very less variation for case I in solar power generation with respect to case II and case III. The monthly average incident solar energy shows the opposite trend as that obtained for efficiency curve. The same pattern has also been observed for maximum output energy in every case. ABC computed monthly average efficiency is achieved for case II, with a maximum value of $10 / 9 \%$. A more flat profile has been obtained foe case III with a value of $8.7 \%$.

$\mathrm{As}, \mathrm{ABC}$, is a behavioural evolutionary technique which is little rare in searching for maximum solar power; so a 
more common method of tracking maximum solar power has also been implemented for the particular three types of cases as considered in this work.

\subsection{Comparative study}

Among all the MPPT algorithms, P\&O method is among the most known technique for tracking maximum solar power $^{[38]}$. The algorithm works on the basis of measured parameters and feedback inputs. On a definite interval of time, the voltage and current generated by the solar module are first subjected to a perturbation. Next, the resulting output power is compared with that of the voltage generated in the previous iteration. The intentional perturbation causes a variation in the solar power generated by the solar module. In the next step, the power is checked with its predefined value; and if the power increases, then the amount of perturbation remains the same in the earlier direction. Finally, when the generated power matches the maximum value of a predefined maximum power, the perturbation is reversed and keeps on decreases for further iterations, while checking with the set value.

However, the result oscillates about the peak value of power corresponding to the algorithm oscillates around. Perturbation size is assumed very small for keeping the power variation within a small range. The implementation of $\mathrm{P} \& \mathrm{O}$ has been made through the following steps as shown below;

1. Generate random solutions $(1,2,3, \ldots . \mathrm{k})$, within the installed constraining range: $0<\beta<90 ; 0<\gamma<90$; Isc $=$ rand $[0,1]$ (Isc max-Iscmin); Voc $=$ rand $[0,1] *($ Vocmax-Vocmin $)$

2. Calculate $\mathrm{V}(\mathrm{k}) ; \mathrm{I}(\mathrm{k})$

3. Calculate $\mathrm{P}(\mathrm{k})=\mathrm{V}(\mathrm{k}) * \mathrm{I}(\mathrm{k})$

4. Calculate $\Delta \mathrm{P}(\mathrm{k})=\mathrm{P}(\mathrm{k})-\mathrm{P}(\mathrm{k}-1)$

5. Check for $\Delta \mathrm{P}(\mathrm{k})>0 ; \Delta \mathrm{V}(\mathrm{k})>0$;

6. Accordingly, $\mathrm{V}(\mathrm{K})$ and $\mathrm{P}(\mathrm{k})$ is updated with decreased or increased module voltage.

The monthly average maximum output energy, when computed by $\mathrm{P} \& \mathrm{O}$ method, has been found to have a similar pattern of variation as that obtained for all the cases when evaluated by ABC.

Further, this has made the ABC algorithm more robust while computing solar power with varying tilt angle.

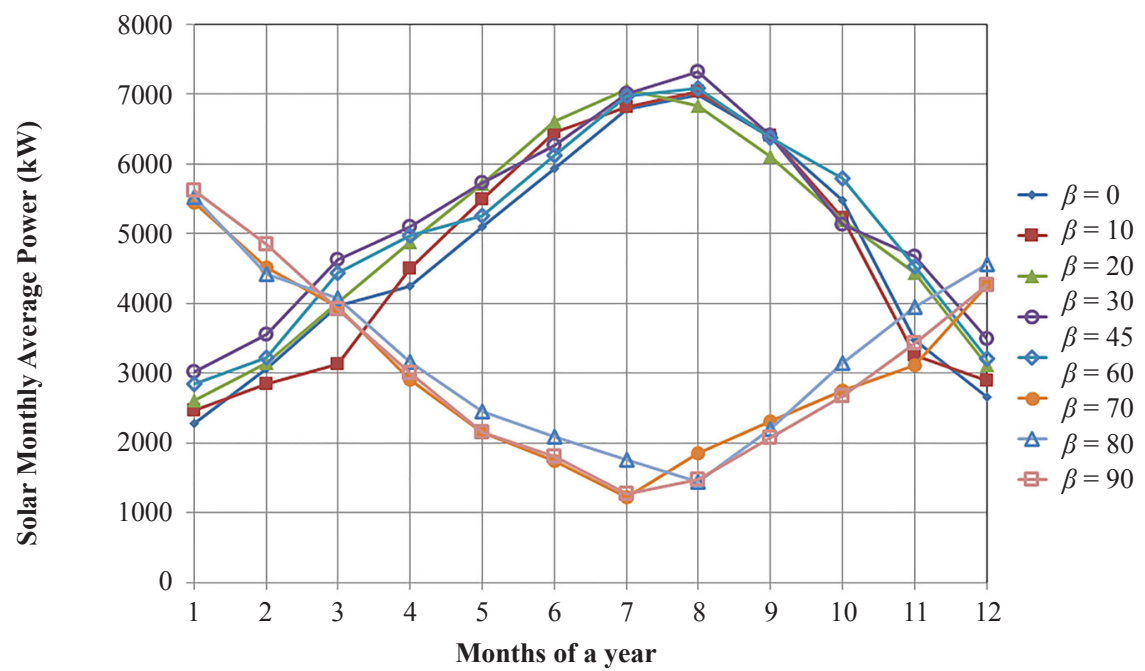

Figure 7. Variation of average monthly solar energy with different tilt angles for Case II as computed by P\&O

Variation of monthly solar energy generated for case II as evaluated by P\&O method is presented in figure 7. Though a similar pattern has been generated by the application of $\mathrm{P} \& \mathrm{O}$ as that generated by $\mathrm{ABC}$; but still the minimum $\&$ maximum power generation achieved by $\mathrm{P} \& \mathrm{O}$ is better than that of ABC. Moreover, the efficiency obtained by the application of $\mathrm{P} \& \mathrm{O}$ method is about $9.2 \%$ in case II, while $7 \%$ for case III.

The maximum and minimum values of solar energy obtained for case II and case III as computed by $\mathrm{ABC}$ and $\mathrm{P} \& \mathrm{O}$ techniques have been depicted in a comparative manner in table II as follows: 
Table 2. Comparative study of $\mathrm{ABC}$ and P\&O Computing Methods

\begin{tabular}{ccccc}
\hline \multicolumn{2}{c}{ ABC Technique } & \multicolumn{2}{c}{ P\&O Technique } \\
\hline & Case II & Case III & Case II & Case III \\
Maximum Solar energy (Tilt angle) & 6.7 & 5.52 & 6.83 & 6.5 \\
Minimum Solar (Tilt angle) & 2.5 & 1.34 & 2.26 & 2.14 \\
\hline
\end{tabular}

\section{Conclusion}

The performance of a PV module has been studied with different varying parameters and conditions. The results can be summarized as the following. The important factors that affect the operational performance of the PV unit are its orientation and tilt angle.

A behavioural evolution-based technique; Artificial Bee Colony has been implemented in locating optimum tilt angle and orientation of PV modules. Maximum average solar energy has been obtained from $20^{\circ}$ to $30^{\circ}$ in both case II and case III. Case studies have been performed on the basis of a typical year of the meteorological conditions for Tripura (India).

Moreover, in analyzing the performance of both techniques, i.e. $\mathrm{ABC}$ and $\mathrm{P} \& \mathrm{O}$ in the present problem; it has been observed that the $\mathrm{P} \& \mathrm{O}$ algorithm yielded better results while computing maximum solar power generation. This can be explained as $\mathrm{P} \& \mathrm{O}$ method is generally used for MPPT; i.e. for maximization problem, so with the variation of tilt angles, the $\mathrm{P} \& \mathrm{O}$ method resulted at a higher value of solar power. While, on the other hand, as $\mathrm{ABC}$ is an evolution-based method, like PSO, AC, GA; which works well for minimization problem; so the minimum values obtained by $\mathrm{ABC}$ was less than $\mathrm{P} \& \mathrm{O}$. However, the efficiency resulted for case II as computed by $\mathrm{ABC}$ was best among other methods as evaluated by both the method.

Therefore it can be inferred that the effects of ambient temperature, solar tilt angle, has to be considered as one of the very important factors in evaluating photovoltaic module output. Further, heuristic techniques can also be implemented in this regard, which can contemplate the uncertainties and approximations of environmental data.

Though the heuristic techniques can envisage the uncertainties and approximations while dealing with renewable energy resources, in consideration with some more parameters and factors different power generating approaches can be adapted, which may lead to better results in order to generate maximum power from solar energy.

\section{References}

[1] Kolnichenko, Georgij I., Yakov V. Tarlakov. Automation of control of electric generators from renewable energy sources in hybrid power supply systems. Journal of Physics: Conference Series. 2020; 1515.

[2] Giwa, Adewale, et al. A comprehensive review on biomass and solar energy for sustainable energy generation in Nigeria. Renewable and Sustainable Energy Reviews. 2017; 69: 620-641.

[3] Mas'ud, Abdullahi Abubakar, et al. Solar energy potentials and benefits in the gulf cooperation council countries: A review of substantial issues. Energies. 2018; 11(2): 372.

[4] Mohammadnia, Ali, et al. Hybrid energy harvesting system to maximize power generation from solar energy. Energy Conversion and Management. 2020; 205: 112352.

[5] Khan, Jibran, Mudassar H. Arsalan. Solar power technologies for sustainable electricity generation-A review. Renewable and Sustainable Energy Reviews. 2016; 55: 414-425.

[6] Rohankar, Nishant, et al. A study of existing solar power policy framework in India for viability of the solar projects perspective. Renewable and Sustainable Energy Reviews. 2016; 56: 510-518.

[7] Logan, Benjamin, Marian Tzolov. Modeling of the electric characteristics of solar cells. APS. 2017; 2017: G1-104.

[8] Chaichan, Miqdam Tariq, Hussein A. Kazem. Environmental conditions and its effect on PV performance. Generating electricity using photovoltaic solar plants in iraq. Springer, Cham; 2018; 83-129.

[9] Järvelä, Markku, Seppo Valkealahti. Ideal operation of a photovoltaic power plant equipped with an energy storage system on electricity market. Applied Sciences. 2017; 7(7): 749.

[10] Liu, Li-qun, et al. Solar energy development in China-a review. Renewable and Sustainable Energy Reviews. 2010; 14(1): 301-311.

[11] Ni, Jian, et al. Modeling and displaying dust accumulation process on solar panel and impacts on photoelectrical conversion in a virtual reality environment. Journal of Advanced Optics and Photonics. 2018; 1(1): 53.

[12] Icel, Yasin, et al. Photovoltaic panel efficiency estimation with artificial neural networks: Samples of adiyaman, malatya, and sanliurfa. International Journal of Photoenergy. 2019.

[13] Mussard, Maxime, Mohamed Amara. Performance of solar photovoltaic modules under arid climatic conditions: A 
review. Solar Energy. 2018; 174: 409-421.

[14] Khan, Rafid Adnan, M. Rezwanul Mahmood, et al. Enhanced energy extraction in an open loop single-axis solar tracking PV system with optimized tracker rotation about tilted axis. Journal of Renewable and Sustainable Energy. 2018; 10(4): 045301.

[15] Safitra, Arrad Ghani, Fifi Hesty Sholihah, et al. Experimental study of slope angle and low E glazing effects on photovoltaic module. IOP Conference Series: Earth and Environmental Science. IOP Publishing; 2018; $105: 1$.

[16] El Khadraoui, Aymen, et al. Thermal behavior of indirect solar dryer: Nocturnal usage of solar air collector with PCM. Journal of cleaner production. 2017; 148: 37-48.

[17] Al Garni, Hassan Z., Anjali Awasthi, et al. Optimal orientation angles for maximizing energy yield for solar PV in Saudi Arabia. Renewable Energy. 2019; 133: 538-550.

[18] Al-Falahi, Monaaf DA, et al. Sizing and modeling of a standalone hybrid renewable energy system. 2016 IEEE 2nd annual southern power electronics conference (SPEC). IEEE. 2016.

[19] Alghamdi, Abdulsalam S., AbuBakr S. Bahaj, et al. Assessment of large scale photovoltaic power generation from carport canopies. Energies. 2017; 10(5): 686.

[20] Gopinathan, K. K., N. B. Maliehe, M. I. Mpholo. A study on the intercepted insolation as a function of slope and azimuth of the surface. Energy. 2007; 32(3): 213-220.

[21] Mohanty, Sthitapragyan, Prashanta Kumar Patra, et al. Prediction and application of solar radiation with soft computing over traditional and conventional approach-A comprehensive review. Renewable and Sustainable Energy Reviews. 2016; 56: 778-796.

[22] Samani, Pouya, et al. Pre-fabricated, environmentally friendly and energy self-sufficient single-family house in Kenya. Journal of Cleaner Production. 2017; 142: 2100-2113.

[23] Li, D. H. W., T. N. T. Lam, et al. Relationship between the total solar radiation on tilted surfaces and the sunshine hours in Hong Kong. Solar energy. 2008; 82(12): 1220-1228.

[24] Guo, Mian, et al. Optimal tilt angle and orientation of photovoltaic modules using HS algorithm in different climates of China. Applied Sciences. 2017; 7(10): 1028.

[25] Chang, Y.-P. An ant direction hybrid differential evolution algorithm in determining the tilt angle for photovoltaic modules. Expert Syst. Appl. 2010; 37: 5415-5422.

[26] Elsheikh, A. H., M. Abd Elaziz. Review on applications of particle swarm optimization in solar energy systems. International Journal of Environmental Science and Technology. 2019; 16(2): 1159-1170.

[27] Safdarian, Farnaz, Mohammad Esmaeil Nazari. Optimal tilt angle and orientation for solar collectors in Iran. 2015 IEEE 10th International Symposium on Diagnostics for Electrical Machines, Power Electronics and Drives (SDEMPED). IEEE. 2015.

[28] Babatunde, A. A., S. Abbasoglu, M. Senol. Analysis of the impact of dust, tilt angle and orientation on performance of PV Plants. Renewable and Sustainable Energy Reviews. 2018; 90: 1017-1026.

[29] Ekanem, Okon Dominic, James O. Onojo. Determination of yearly fixed optimal tilt angle for flat-plate photovoltaic modules based on perez transposition model. American Journal of Software Engineering and Applications. 2017; 6(3): $80-84$.

[30] Ghazouani, Karima, et al. Thermal analysis of linear solar concentrator for indirect steam generation. Energy Procedia. 2019; 162: 136-145.

[31] Som, T., R. Pathak. Maximum solar power generation through optimization of tilt solar angles of solar panels by heuristic technique. International Journal of Innovative Research in Electrical, Electronics, Instrumentation and Control Engineering. 2015; 10.

[32] Guo, Mian, et al. Optimal tilt angle and orientation of photovoltaic modules using HS algorithm in different climates of China. Applied Sciences. 2017; 7(10): 1028.

[33] Rajput, Pramod, et al. Life cycle assessment of the $3.2 \mathrm{~kW}$ cadmium telluride (CdTe) photovoltaic system in composite climate of India. Solar Energy. 2018; 159: 415-422.

[34] Karaboga, Dervis, Bahriye Basturk. Artificial bee colony (ABC) optimization algorithm for solving constrained optimization problems. International fuzzy systems association world congress. Springer, Berlin, Heidelberg; 2007.

[35] Yao, Jing, Ju-hou He. Load balancing strategy of cloud computing based on artificial bee algorithm. 20128 th International conference on computing technology and information management (NCM and ICNIT). IEEE. $2012 ; 1$.

[36] Adaryani, M. Rezaei, A. Karami. Artificial bee colony algorithm for solving multi-objective optimal power flow problem. International Journal of Electrical Power \& Energy Systems. 2013; 53: 219-230.

[37] Bhattacharya, Tanima, Ajoy K., et al. Effects of ambient temperature and wind speed on performance of monocrystalline solar photovoltaic module in Tripura, India. Journal of Solar Energy. 2014.

[38] Nedumgatt, Jacob James, et al. Perturb and observe MPPT algorithm for solar PV systems-modeling and simulation. 2011 Annual IEEE India Conference. IEEE. 2011. 